\title{
POESÍA INFANTIL DESDE LOS VALORES PARA UNA SOCIEDAD BILINGÜE MEDIANTE LAS TIC
}

\section{CHILDREN'S POETRY FROM THE VALUES FOR A BILINGUAL SOCIETY THROUGH ICT}

\author{
María Amor Martín Fernández*, Eloísa Reche Urbano, \\ Begoña E. Sampedro Requena, Belén Quintero Ordoñez, \\ Ana Muñoz Mallen \\ * fe1mafem@uco.es
}

\begin{abstract}
Resumen
La experiencia que se presenta se ha desarrollado a lo largo del curso académico 2016-2017, con el alumnado de primero, segundo y cuarto curso (mención de Lengua Extranjera) del Grado en Educación Primaria de los Centros de la Universidad de Córdoba en los que se cursa esta titulación, en diversas asignaturas correspondientes a las áreas de "Didáctica de la Lengua y la Literatura", "Tecnología educativa", "Didáctica y Organización Escolar" y "Filología inglesa". Su objeto central ha sido la poesía infantil; por una parte, la poesía folclórica o tradicional, patrimonio poético infantil de tradición oral que corre el peligro de perderse y, por otra, la poesía de autores de la literatura española, hecha especialmente para la infancia, atendiendo principalmente a dos aspectos: los valores humanos que en ellas se contienen y su presentación en formatos acordes a los medios de comunicación actuales. Para ello, se ha diseñado y editado material multimedia educativo, dirigido al alumnado de Educación Primaria, que contribuya a la recuperación y difusión de la lírica infantil, de una manera original, atractiva y lúdica, y a su puesta en valor en una sociedad mediada por la tecnología y bilingüe. Los resultados académicos revelan la eficacia de este tipo de propuestas, así como la satisfacción e implicación del alumnado con ello. La experiencia confirma que este tipo de prácticas que evidencian la funcionalidad de los aprendizajes y la relación de la teoría con la práctica favorecen la implicación del alumnado en su proceso formativo, les sensibilizan del compromiso social de la institución universitaria con la difusión de la literatura y, con ello, de la cultura española, como elemento de transferencia de conocimiento en una sociedad multilingüe e intercultural como en la que vivimos.
\end{abstract}

Palabras clave: poesía infantil; innovación educativa; material multimedia; educación en valores; bilingüismo.

\begin{abstract}
The experience presented has been carried out throughout the 2016-2017 academic year, with the students of the first, second and fourth year (Foreign Language mention) in the Degree of Primary Education from the Centers at the University of Córdoba where this bachelor's degree is offered, in different subjects which cover the areas of "Language Didactics and Literature", "Educative Technology", "Didactics and School Organization" and "English Philology". Its main object has been children's poetry; on the one hand, the traditional and folk poetry, child poetic heritage of oral tradition that is at risk of being lost and, on the other hand, poetry by authors of Spanish literature, written specially for children, focusing attention mainly on two aspects: human values that this poetry contains, and its presentation in formats according to today's media. For that purpose, it has been designed and edited multimedia educational material, addressed to Primary Education students, which contributes to the recovery and dissemination of infant lyric, in an original, appealing and entertaining way, and to its value in a bilingual society mediated by technology. The academic achievement reveals the efficiency of this type of proposals as well as the student's contentment and involvement in it. The experience proves that this type of practice that shows the learning functionality and the relation between theory and practice promotes the students' involvement in their learning process, raises awareness about the social commitment of the university institution to the dissemination of literature and, thus, of the Spanish culture, as an element of knowledge transfer in a multilingual and intercultural society like that where we live.

Keywords: children's poetry; educational innovation; multimedia material; values education; bilingualism.
\end{abstract}

\section{INTRODUCCIÓN}

Es una realidad incuestionable que las tecnologías se han convertido en recursos que conviven en el quehacer diario de las personas (ocio, trabajo, comunicación, etc.); también lo es que se han incorporado con fuerza en las aulas, tanto universitarias como en otras etapas educativas, para favorecer el aprendizaje del alumnado y convertirse en elementos indispensables en la formación, inicial y permanente, de cualquier docente (Durán y Vega, 2013; Fernández-Batanero y Torres-González, 2015).

Las tendencias apuntan además, a medio y corto plazo, a la implementación del denominado Deeper Learning, como se recoge en el Informe Horizon 2016 (Johnson et al., 2016); es decir, el dominio de contenidos que potencien en el alumnado un pensamiento crítico y reflexivo a través de la resolución de problemas con carácter colaborativo generando aprendizaje. Este efecto puede ser promovido mediante actividades académicamente dirigidas (Marín y Reche, 2012) para fomentar un aprendizaje profundo. 
Por otra parte, es preciso propiciar la incorporación de recursos didácticos motivadores y atractivos para el alumnado, usando para ello el mundo de la imagen y las tecnologías (Sánchez, 2010) y la palabra para desarrollar un aprendizaje cooperativo, donde la transversalidad desempeñe un papel importante.

A ello hay que unir la necesidad de que "el fenómeno de la globalización mundial atienda a una educación plurilingüe que potencie modelos de enseñanza destinados a educar en la tolerancia lingüística" (Bazo, D’Angelo y Valdés, 2010, p.4). En 2002, el Marco Común Europeo de Referencia para las Lenguas (MCERL) presta especial atención al enfoque plurilingüe al describir entre otros aspectos que:

El hecho de que conforme se expande la experiencia lingüística de un individuo en los entornos culturales de una lengua, desde el lenguaje familiar hasta el de la sociedad en general, y después hasta las lenguas de otros pueblos (ya sean aprendidas en la escuela o en la universidad, o por experiencia directa), el individuo no guarda estas lenguas y culturas en compartimentos mentales estrictamente separados, sino que desarrolla una competencia comunicativa a la que atribuyen todos los conocimientos y la experiencias lingüísticas y en la que las lenguas se relacionan entre sí e interactúan. (MCERL, p. 4).

Las sociedades actuales favorecen contextos en los que conviven personas de diferentes culturas e identidades, siendo la educación intercultural el vehículo apropiado para desarrollar valores humanos que faciliten su interrelación.

En este contexto, el texto literario, lejos de sentirse desbancado en la sociedad mediática y globalizada, aporta unas "posibilidades expresivas y comunicativas a las que resultaría imposible acceder mediante el uso exclusivo de los textos y recursos convencionales y no literarios" (Mendoza, 2006, p. 293) ya que, en sí misma, la literatura es expresión universal de la experiencia humana. Y de ella, junto a la poesía de autores relevantes que han escrito para la infancia, se ha elegido la poesía popular infantil, de transmisión oral, esa, en palabras de López Tames (1990), que "se refiere al «corpus» tradicional de poesía popular que tiene al niño como protagonista y objeto" (p. 157) y que ha visto rota su cadena de transmisión por los cambios en el actual contexto social: nuevas formas de trabajo y ocio, en especial de los niños, nuevos modos y espacios de relaciones sociales, nuevos medios de comunicación, nuevos juegos infantiles vinculados en gran medida a dispositivos tecnológicos, entre otros. En este escenario:

Volver a tener una tradición oral pujante es hoy un sueño casi imposible. Nuestra responsabilidad, como herederos de este importantísimo acervo cultural, debe llevarnos, al menos, a intentar que no se pierda lo que aún queda y, quizá, la única manera de hacerlo es fijar por escrito algo que, sin embargo, nació para ser dicho, cantado y escuchado (Cerrillo y Sánchez, 2012, p. 24).

Para colaborar en ello, esta experiencia plantea la alianza de la poesía con los nuevos escenarios de la Sociedad de la Información y la Comunicación y sus recursos tecnológicos.

\section{OBJETIVOS}

La poesía infantil es el objeto central de esta propuesta. Por una parte, la poesía folclórica, patrimonio poético de tradición oral y, por otra, la poesía de autores de la literatura española hecha especialmente para la infancia y su presentación a través de nuevos medios de comunicación, que colaboren a evitar la ruptura en su transmisión y difusión.

Para ello, se pretende:

1. Editar material multimedia que pueda ser utilizado en las aulas de la etapa de Educación Primaria, para contribuir a recuperar, transmitir y difundir la poesía infantil.

2. Colaborar en el conocimiento y difusión, desde las aulas de la Enseñanza Obligatoria (Infantil y Primaria), de la poesía popular infantil, patrimonio de nuestra cultura.

3. Iniciar al alumnado del Grado en Educación Primaria en la creación de textos poéticos infantiles y en la adaptación y traducción a una segunda lengua de poemas infantiles.

4. Mostrar a la sociedad los valores humanos contenidos en estas obras y la importancia de la experiencia estética.

5. Formar al alumnado participante del Grado en las competencias transversales (TIC, dominio de una segunda lengua extranjera y emprendimiento) a través de actividades académicamente dirigidas multidisciplinares.

6. Sensibilizar a los futuros docentes en el compromiso social de la Universidad con la difusión de la cultura española, como elemento de transferencia de conocimiento.

\section{Metodología}

Esta experiencia se ha desarrollado a lo largo del curso académico 2016-2017, con el alumnado del Grado en Educación Primaria de los dos centros de la Universidad de Córdoba en los que se imparte esta titulación en las siguientes asignaturas:

- Música, Plástica y Dramatización como Recursos Didácticos en Lengua Extranjera (Inglés) (a partir de ahora MPDI), impartida en $4^{\circ}$ curso, primer cuatrimestre, en el Centro de Magisterio "Sagrado Corazón", adscrito a la Universidad de Córdoba. 
- Métodos de Investigación Educativa y Aplicaciones de las TIC (a partir de ahora METTIC), impartida en $1{ }^{\text {er }}$ curso, segundo cuatrimestre, tanto en el Itinerario Bilingüe como en otras dos líneas más, en la Facultad de Ciencias de la Educación.

- Convivencia Escolar y Cultura de Paz en Educación Primaria (a partir de ahora CCEE), impartida en $2^{\circ}$ curso, segundo cuatrimestre, en el Centro de Magisterio "Sagrado Corazón".

- Formación Literatura y Literatura Infantil (a partir de ahora FORMLIT), impartida en $2^{\circ}$ curso, segundo cuatrimestre, a dos grupos aula (grupo 1 y grupo 2), en el Centro de Magisterio "Sagrado Corazón".

- Educación Mediática y Aplicaciones Didácticas de las TIC (a partir de ahora EMADT), impartida en $2^{\circ}$ curso, segundo cuatrimestre, en el Centro de Magisterio "Sagrado Corazón".

La experiencia se inserta así, tanto en el Centro de Magisterio "Sagrado Corazón" como en la Facultad de Ciencias de la Educación, en el marco de las características propias del proceso de enseñanza-aprendizaje del alumnado y de su formación en el paquete competencial de las asignaturas.

La metodología seguida, en las materias citadas, ha sido variada en función de las necesidades y especificidades de cada una.

En la búsqueda de dar cobertura a las diferentes finalidades, objetivos y momentos de actuación, se ha recurrido a las clases teóricas, basadas en la técnica de la lección magistral; a los talleres y laboratorio de poseía infantil; a las clases prácticas para la traducción de los textos (español a inglés), para la utilización de herramientas informáticas y para la búsqueda de información de carácter virtual; a sesiones de trabajo de carácter individual y grupal, así como a las tutorías de asesoramiento y seguimiento. Todo ello puesto que es necesario desarrollar, de manera eficaz, tanto el corpus teórico-práctico de la materia, como las acciones de innovación.

Por su parte, la lección magistral ha tenido como finalidad poder dotar al estudiante de unos conocimientos, habilidades y destrezas básicas y necesarias, para adquirir o desarrollar, en parte, las competencias que se establecen en las materias. Con la misma finalidad, las sesiones prácticas se han llevado a cabo después de las lecciones magistrales, dando continuidad a los conceptos ya explicados, favoreciendo la aplicabilidad en los procedimientos y el progreso de las competencias. Entre las técnicas empleadas, a nivel general destacan las sesiones dedicadas a la búsqueda y selección de información, como base para desarrollar el material solicitado y las sesiones para el manejo de herramientas tecnológicas digitales (Blogger, Plotagon Education y Movie Maker o iMovie), que han servido de soporte y medio de difusión del material multimedia diseñado y editado.

En esta misma línea y de manera complementaria, la variedad de talleres ha favorecido la interactividad, el contractar opiniones y la construcción del aprendizaje de manera grupal. También el laboratorio de lírica infantil, diseñado para iniciar al alumnado en las posibilidades de la palabra y en el oficio de escribir, tiene como objetivo fundamental desarrollo de la creatividad literaria y dotar de los aprendizajes necesarios para la creación de poemas infantiles.

Por último, las tutorías de asesoramiento y seguimiento del trabajo del alumnado, en su modalidad presencial, así como virtual a través de correo electrónico, han hecho posible la atención personalizada de los y las estudiantes o del grupo de trabajo en un ambiente con garantías de privacidad y tranquilidad de la comunicación (De Miguel, 2006).

Con la intención de conocer la funcionalidad y consecución de los objetivos planteados en la innovación durante el desarrollo de las asignaturas, se han abordado una serie de elementos propios de los estudios que, a continuación, se describen.

a) Objetivos

Los objetivos de investigación diseñados han sido los siguientes:

- Establecer el nivel de satisfacción que tiene el alumnado, de cuarto del Grado de Educación Primaria (mención Lengua Extranjera) del Centro de Magisterio "Sagrado Corazón”, con la experiencia formativa realizada en la asignatura MPDI.

- Determinar el grado de valoración que realiza el alumnado, de primero del Grado en Educación Primaria de la Facultad de Ciencias de la Educación, de tres de las cuatro líneas (A, B y D), de las modalidades de enseñanza empleadas en la asignatura METTTC que más le han facilitado su formación.

- Determinar el grado de valoración que realiza el alumnado, de segundo del Grado en Educación Primaria del Centro de Magisterio "Sagrado Corazón”, de las modalidades de enseñanza empleadas en las asignaturas CCEE, FORMLIT y EMADT que más le han ayudado en su aprendizaje.

- Conocer el grado de estimación que poseen los y las estudiantes sobre determinados aspectos de su aprendizaje, en las materias METTIC, CCEE, FORMLIT y EMADT.

- Averiguar la percepción que tiene el alumnado participante sobre el grado de implicación (motivación y/o participación) que han mostrado en la consecución de los objetivos de las asignaturas METTIC, CCEE, FORMLIT y EMADT. 


\section{b) Diseño}

Para efectuar este estudio es necesario determinar cuál será el patrón o guía en el que basarlo, al cual se le denomina paradigma de investigación. En este caso es la composición de dos, considerando la existencia de un acuerdo de tres corrientes en la investigación educativa (Sabariego, 2012); por un lado, el paradigma positivista vinculado con un carácter más cuantitativo y que explicará la valoraciones, estimaciones y percepciones que realizan los estudiantes sobre su formación, aprendizaje e implicación en las asignaturas y, por ende, en la implementación de la experiencia. Por otro, el paradigma socio-critico asociado a una metodología, preponderantemente cualitativa, en la búsqueda de respuesta de problemas o transformaciones sociales; en otros términos, comprender la situación de implementar acciones formativas innovadoras en el aula universitaria desde la visión del alumnado.

Según el grado de intervención y considerando la clasificación de Sabariego y Bisquerra (2012), esta atiende a una modalidad de corte descriptivo, ex post facto, en la que el diseño obedece a la experimentalización que trata de optimizar, a través de la detección de las condiciones previas, las percepciones de los estudiantes y sus valoraciones en relación a su aprendizaje efectivo en las aulas universitarias y, además, a la implementación de acciones formativas de innovación donde ellos serán participes con su implicación (creación y difusión de materiales multimedia).

c) Muestra

Como se ha mencionado, la muestra está conformada, por un lado, por los estudiantes de primero del Grado en Educación Primaria de la Facultad de Ciencias de la Educación, de tres de las cuatro líneas (A, B y D) y, por otro, el alumnado de segundo del Grado en Educación Primaria (grupo 1 y grupo 2) y de cuarto de la misma Titulación, mención Lengua Extranjera, matriculados en el Centro de Magisterio "Sagrado Corazón" que se ofertan en la Universidad de Córdoba.

Con respecto al alumnado de la Facultad de Ciencias de la Educación, las características definitorias de estos, son las siguientes:

- En total son 176 estudiantes, distribuidos el 30,7\% (N=54) en la línea A o bilingüe; el 38,6\% (N=68) en la línea $B$ y; el 30,7\% (N=54) en la línea $\mathrm{D}$.

- $\quad$ El 67\%(N=118) son mujeres y, el 33\% (N=58) hombres.

- La edad mínima de los participantes es 18 y la máxima 42 años, siendo la media de M=19,64 (DS=3.193).

Por su parte, las características del alumnado del Centro de Magisterio son:

- En total son 117 estudiantes, distribuidos el 47\% (N=55) en grupo 1 de segundo; el 39,3\% (N=46) en grupo 2 de segundo y el 13,7\% (N=16) en cuarto, mención Lengua extranjera.

- $\quad$ El 50,4\%(N=59) son mujeres y, el 49,6\% (N=58) hombres

- La edad mínima de los participantes es 19 y la máxima 36 años, siendo la media de M=21,33 (DS=3.337).

\section{d) Instrumentos}

El instrumento de recogida de información utilizado ha sido el cuestionario, al facilitar este "traducir variables empíricas sobre las que se desea información, en preguntas concretas capaces de suscitar respuestas fiables, válidas y susceptibles de ser cuantificadas de un amplio grupo de participantes" (González, Marín y Martín, 2015, p. 40).

En función de las características propias de las diversas materias que participan, se han creado, ad hoc, diferentes herramientas, consensuadas por el profesorado participante en el mismo.

Para la asignatura MPDI, el cuestionario quedó constituido por cinco bloques: el primero recoge los ítems de las variables sociodemográficas (sexo y edad), el segundo de ellos formado por 9 preguntas escalares (con valores del $1=$ muy insatisfecho a $5=$ muy satisfecho), el tercer bloque lo conforma una pregunta de carácter abierto, en la cual se dio la posibilidad de explicar las razones de satisfacción o insatisfacción de los ítems valorados en el primer bloque. El cuarto bloque lo constituye una pregunta de reflexión sobre posibles cambios en algunos de los pasos, así como en la metodología empleada para afrontar la práctica. Y en el último bloque, su apreciación sobre el grado de adquisición de las competencias, una vez finalizada la práctica.

Con respecto a la materia METTIC, el instrumento empleado para obtener los datos se distribuyó en cuatro partes bien diferenciadas. La primera de ellas constituyen los ítems de las variables sociodemográficas como el sexo, la edad y la línea en la que se encuentra adscrita la muestra participante. Un segundo apartado recoge once ítems sobre el grado de valoración de las modalidades de enseñanza respecto a la validez para el proceso de formación, concretamente, cuatro para las lecciones magistrales de cada uno de los temas o aspectos teóricos (introducción a la sociedad de la información y búsquedas en Internet, el entorno Google y sus aplicaciones, los Blogs y las comunidades en red); seis para las sesiones prácticas uno por cada aspectos práctico abordado (búsqueda efectiva y eficiente de información, Google y su entorno, la aplicación Blogger, la aplicación Plotagon Education, el programa Movie Maker, la aplicación Google+) y, finalmente, un ítem correspondiente 
a la modalidad de las tutorías o asistencia grupal sobre aspectos puntuales. La escala elegida para este apartado ha sido Likert de cuatro opciones, donde 1 significaba nada; 2 es poco; 3 es bastante; $y, 4$ es mucho.

El tercer apartado agrupa cinco ítems sobre la estimación de algunos aspectos relacionados con el aprendizaje: tres ítems sobre la mejora de la competencia digital, informacional y tecnológica o informática, un ítem sobre gestión personal del tiempo y un último ítem sobre la atención en las clases teóricas. Atendiendo a la formulación de este grupo, siendo afirmaciones o sentencias ("La gestión realizada del tiempo ha logrado una obtención progresiva de las tareas propuestas"), se ha empleado para su medición una escala Likert de cinco opciones, donde 1 significaba "totalmente en desacuerdo" y 5 es "totalmente de acuerdo".

Por último, el cuarto apartado agrupa trece ítems, cada uno de los cuales corresponde a los fines pedagógicos planificados tanto para la asignatura como para las acciones de innovación; considerando que esta dimensión intenta medir la percepción del sujeto relativa a su implicación (motivación y/o participación) en la consecución de los objetivos, la escala tipo Likert ha sido de cuatro opciones, donde 1 significaba nada; 2 es poco; 3 es bastante; y, 4 es mucho.

Por su parte, el instrumento utilizado para recabar información del alumnado sobre la realización de la práctica interdisciplinar, en el marco de las asignaturas CCEE, FORMLIT y EMADT, quedó conformado por tres bloques.

El primero de ellos con los ítems de las variables de identificación de la muestra: el sexo, la edad y el grupo aula.

El segundo bloque, a su vez, se estructuró en dos apartados. Uno sobre la metodología utilizada, compuesta por 9 preguntas escalares que valoraban el grado de utilidad de los diversos métodos docentes (lecciones magistrales, talleres, laboratorio de poesía infantil, sesiones para la revisión de la selección y adecuación de los poemas infantiles, sesiones de consultas y de trabajo de carácter presencial) y, a continuación, otro con 7 cuestiones, de carácter escalar, que recoge la apreciación del alumnado sobre el nivel de logro de los objetivos de la experiencia, teniendo en cuenta el proceso y la realización del material audiovisual educativo solicitado.

Para concluir, el cuestionario se cerró con 9 preguntas: 7 de tipo escalar sobre el grado de implicación de los estudiantes en las distintas fases de elaboración de esta práctica y una pregunta de carácter abierto, en la cual se ofrecía la posibilidad de comentar los aspectos que mejorarían de su participación, en función de la valoración que otorgasen con anterioridad.

Las opciones de respuesta para las preguntas de tipo escalar, en este instrumento, oscilaron con valores del $1=\mathrm{Nada}$, $2=$ Poco, $3=$ Algo, $4=$ Bastante y $5=$ Mucho.

Para los diversos cuestionarios creados, no se han ejecutado análisis sobre la fiabilidad y validez del instrumento, dado que el estudio busca una descripción y exploración de la realidad educativa a la que va dirigida, más que una investigación de profundidad.

e) Estrategias de análisis de datos

El análisis de los datos recogidos tras la aplicación del cuestionario fue de carácter eminentemente cuantitativo, efectuando análisis descriptivos básicos de tendencia central y medidas de dispersión de las diversas variables con el programa estadístico SPSS (versión 18) y para las aportaciones de tipo cualitativo, el tratamiento de la información se realizó mediante un procedimiento deductivo.

\section{DESCRIPCIÓN}

La experiencia educativa se ha llevado a cabo en varias fases. Una primera de coordinación de los docentes de los centros y materias implicadas para concretar la secuenciación de los contenidos y talleres, especificar las horas lectivas necesarias para la planificación y la edición del material multimedia, en función de las características y posibilidades de cada asignatura, así como, la creación de los diversos guiones de las prácticas, en los cuales se recogieron los distintos aspectos que se debían alcanzar, el cronograma del trabajo del alumnado para el desarrollo del mismo, el modo de entrega y la ponderación en la nota global.

Además, se acordaron los instrumentos de evaluación del material editado y los instrumentos de recogida de información acerca de los distintos matices de la experiencia. Como se comentó en el apartado anterior, se optó por un cuestionario, ad hoc, de satisfacción del alumnado con la experiencia en cuanto a la metodología, el logro de los objetivos de la experiencia y su nivel de implicación, adaptado para cada asignatura.

Comenzado el curso, se procedió a la implementación de las prácticas en las diversas materias. En el primer cuatrimestre, se formalizó para MPDI, con el alumnado de cuarto curso, mención Lengua Extranjera, en el Centro de Magisterio "Sagrado Corazón" para una de las actividades. En el segundo cuatrimestre, por un lado, se estableció en METTIC, con el alumnado de primer curso, en la Facultad de Ciencias de la Educación, como eje temático para la edición de las prácticas asociadas con la tecnología educativa y, por otro lado, como práctica interdisciplinar de CCEE, FORMLIT y EMADT, con el alumnado de segundo curso, en el Centro de Magisterio "Sagrado Corazón". Todas ellas, cerraron su intervención con la implementación del cuestionario, dirigido al alumnado participante. 
En una última fase, se procedió con la evaluación del logro de las competencias, mediante los instrumentos de valoración creados para cada materia y al volcado de los datos de los cuestionarios, el tratamiento y el análisis de los resultados obtenidos con el objetivo de valorar la experiencia.

\subsection{DESARROLLO DE LA EXPERIENCIA EN LA ASIGNATURA "MPDI"}

Atendiendo a los objetivos del proyecto, el alumnado elaboró un vídeo educativo basado en imágenes fijas, montado con el programa Movie Maker, en el cual se presentaba la adaptación y traducción de un texto literario en castellano a la lengua inglesa.

La práctica se llevó a cabo en un periodo de tres semanas, secuenciadas en varias sesiones. La primera de ellas se inició con la selección y reparto de 15 poemas entre el alumnado. A continuación, de manera individual se realizó una lectura del texto literario en español y se profundizó en el contenido de este. Una vez analizado, lo adaptaron y lo tradujeron movilizando los conocimientos iniciales, sin consultar el vocabulario en el diccionario en primera instancia.

Concluidas las traducciones, la docente revisó la corrección de las adaptaciones y la redacción de cada uno de los textos para pasar a una sesión reescritura, en la cual se pudo hacer uso del diccionario online. Finalizada esta, se procedió a una segunda revisión para validar y conformar el texto definitivo. Posteriormente, en una sesión de carácter grupal se analizaron las incidencias del proceso y la revisión conjunta de los errores detectados.

Finalizada esta primera parte del trabajo, el alumnado reflexionó sobre cómo iban a dramatizar sus textos literarios: seleccionar los gestos, lenguaje corporal para expresar sentimientos, concretar el vestuario y los objetos necesarios para narrar audiovisualmente los poemas.

Una vez la profesora les dio el visto bueno, el alumnado grabó los vídeos utilizando, con posterioridad, el programa Movie Maker para su montaje. La entrega del material se formalizó a través de la plataforma UCOMoodle. En la última sesión, se visualizaron algunos de los vídeos para su análisis y se recabó información sobre la opinión del alumnado acerca de la metodología, aprendizajes e implicación del alumnado en el proceso de elaboración y edición del material.

Para proceder a la evaluación del logro de competencias se utilizó como instrumento la escala de valoración. Los ítems que conforman la escala de valoración fueron:

- Las destrezas orales fueron evaluadas en un 50\% y comprendieron los siguientes elementos: adecuación (creatividad, originalidad y motivación), coherencia y cohesión (estrategias comunicativas), pronunciación y entonación, riqueza gramatical y propiedad, y riqueza léxica y propiedad.

- Las destrezas escritas fueron evaluadas en un 50\% y comprendieron los siguientes elementos: adecuación, cohesión y coherencia (estrategias de escritura), riqueza gramatical y precisión, y riqueza léxica y precisión.

\subsection{DESARROLLO DE LA EXPERIENCIA EN LA ASIGNATURA “METTIC”}

La intervención se implementó a lo largo de cinco semanas repartida en varias sesiones, cada una de las cuales abordaba un aspecto, tanto de la materia a impartir en la asignatura de "Métodos de investigación educativa y aplicaciones de las TIC", (de carácter básico, ofertada en la FCCE), como del proyecto de innovación. La distribución fue de cinco sesiones de 90 minutos y cinco de 60 minutos con el gran grupo, y 15 sesiones de 60 minutos con grupos medianos (a cada uno de ellos le correspondieron cinco). Todas las líneas (A o bilingüe, B y C) han tenido la misma temporalidad y disposición debido al grado de experimentación de la asignatura. Asimismo, se buscaron tres horas fuera del horario de la asignatura para tutorías concretas con aquellos pequeños grupos que las necesitaran.

El primer día de comienzo de la asignatura, se muestra el programa oficial de la misma mediante una presentación con la aplicación "Genial.ly". En ella se expone que la materia forma parte de un proyecto de innovación, dentro de la convocatoria del Plan de Innovación para el Curso 2016/2017 de la Universidad de Córdoba, en la modalidad 2. Programa de Formación para la innovación Docente. Debido a esto se le expone al alumnado la siguiente situación:

En un colegio de la etapa de Primaria de Andalucía se han realizado unas pruebas a todo el alumnado del centro para diagnosticar el conocimiento en las asignaturas instrumentales (Lengua Castellana y Literatura, Matemáticas y Lengua Extranjera); el resultado de dicho estudio o investigación ha arrojado un nivel bajo en la competencia literaria (referida al hábito lector, a la capacidad de disfrutar con textos concebidos con una finalidad artística o estética, a las habilidades y conocimientos relacionados con la intertextualidad) y a la competencia semiológica, (relacionada con los saberes necesarios para producir e interpretar mensajes integrados por códigos diversos o que se transmiten por canales y soportes diferentes a los exclusivamente lingüísticos) taly como expresa el Anexo I de la Orden 17 de marzo de 2015 de Andalucía en el área de Lengua Castellana y Literatura. Se nos solicita desde esta institución escolar la producción de materiales audiovisuales relacionados con la literatura infantil, en concreto, con la de tradición oral, para la mejora de este aprendizaje y el progreso de la competencia literaria y semiológica. Dichos materiales se difundirán en la comunidad en red del colegio.

Esta situación es el condicionante de acción de la asignatura, dado que se parte de los resultados extraídos de una investigación diagnóstica sobre el aprendizaje y el desarrollo de las competencias del alumnado de la etapa de Educación Primaria. En este sentido, el alumnado será el encargado de producir y elaborar materiales audiovisuales para ayudar a los docentes de este centro. Por este motivo, los contenidos teóricos y prácticos a abordar durante estas cinco semanas fueron: 
- Contenidos teóricos: Introducción a la sociedad de la información y búsquedas en Internet; El entorno Google; Los Blogs como recurso colaborativo y de difusión; Las comunidades en red como recurso para compartir información y conocimientos de carácter académico y científico.

- Contenidos prácticos: Búsquedas eficaces y eficientes de información; La aplicación Drive y Blogger dentro del entorno de Google; La aplicación Plotagon Education para producir cortos; El programa Movie Maker para producir videos; Google+ como aplicación de Google para crear una comunidad en red.

Inicialmente se emplea la modalidad de lección magistral para comenzar con la introducción a la sociedad de la información y búsquedas en Internet. Se insiste en la relevancia de emplear estrategias de búsqueda de información eficaces en este nuevo escenario y se reflexiona y debate con el alumnado la apropiación indebida de imágenes, audio, vídeos, textos, el plagio y las repercusiones y perjuicios académicos y sociales de este, la importancia de citar adecuadamente, etc. Se les hace una breve conclusión de la importancia de estos conocimientos como usuarios de tecnología y como futuros docentes, así como de las repercusiones de no dominar estos aspectos.

Seguidamente, se les solicita en los grupos medianos que constituyan equipo de trabajo (mínimo tres y máximo cinco miembros), explicando mediante la modalidad de sesión práctica las sintaxis de búsqueda u operadores booleanos, las bases de datos académicas y científicas y la biblioteca digital de la Universidad de Córdoba. Se le presenta la primera tarea a realizar, seis búsquedas individuales sobre "literatura infantil, en concreto, la tradición oral", temática base de las acciones de innovación, recalcando la obligatoriedad de una opción científica (artículo de investigación), una opción académica (significado o normativa sobre competencia literaria y semiología) y un recurso didáctico (juego o aplicación).

A continuación, se les enseña a través de la lección magistral el entorno Google, de manera más concreta, la búsqueda de imágenes, videos, libros, etc., con especial hincapié en Google Académico y en la aplicación Drive, por su carácter colaborativo, pedagógico e investigador.

Las siguientes sesiones tratan sobre los Blogs, tanto a nivel teórico (definición, aspectos y estructuras características, y los beneficios para el ámbito educativo) como práctico mediante la aplicación Blogger. Algún aspecto reseñable de estas clases es la diversidad de conocimientos de estos recursos educativos. El tanteo observado es que el $45 \%$ habían realizado previamente un blog (la mayoría con Blogger y unos pocos con WordPress) y los restantes no habían tenido ningún contacto, ni académico ni personal, con esta herramienta (es necesario aclarar que los porcentajes varían según líneas, sin embargo la proporción es muy similar en B y D).

En este momento, se presenta la segunda tarea, que consiste en la elaboración de un blog grupal, cuyo tema central es la poesía infantil y la tradición oral, y sus requisitos mínimos: tener varios gadget, diseño y formato trabajado, varios recursos audiovisuales, distintas páginas y entradas, hipervínculos, e imágenes debidamente citadas y proporcionando la autoría de estos materiales al autor correspondiente.

Para ello, se explica la aplicación Plotagon Education. En concreto, se trabajaron los requisitos del sistema operativo de los ordenadores para su instalación, observaciones de la versión trial para 30 días (dado que a día de hoy no tiene licencia gratuita), las modalidades: normal y específica de educación, la interfaz del programa, los characters o personajes, y los plots o creación de cortos: personajes, escenas, escenarios, diálogos, música de fondo, lenguaje no verbal de los personajes, etc. A la línea A se les pide obligatoriamente diseñar, con esta aplicación, un recurso audiovisual basado en la temática de la poesía de tradición oral, mientras que a las restantes líneas (B y D) solo se les recomendó.

Asimismo, mediante sesiones prácticas se presentó el programa Movie Maker, gratuito para la edición de videos; entre los contenidos prácticos explicados se encuentran: requisitos del sistema operativo de los ordenadores para su instalación, interfaz del software, animaciones, inclusión de pistas narrativas, de audio, clip de videos, imágenes fijas, etc. y combinación de elementos. Posteriormente se explicó, mediante el seguimiento en la modalidad de tutorías o asistencia grupal, cómo se podía obtener el video, ya fuera con Plotagon Education o con Movie Maker, y su publicación en la aplicación YouTube del entorno Google.

Durante diversas sesiones, los estudiantes trabajaron en la segunda tarea diseñando el blog, los recursos audiovisuales y seleccionando la información que recogían en el mismo. Para estos períodos se aplica la modalidad de tutorías o asistencia grupal sobre aspectos puntuales, efectuando un seguimiento de la tarea a los 12 equipos formados para abordar esta actividad en la línea A (16 equipos en la línea B y 11 grupos en la línea D). Es interesante observar la mecánica que emplearon para el trabajo en grupo. La mayoría, aproximadamente el $85 \%$, se repartieron las actividades, haciéndose expertos en algún aspecto y recayendo en un solo sujeto el liderazgo y la responsabilidad en los plazos. Existen aspectos reseñables, como por ejemplo, la necesidad continua de revisión de las actividades, la preocupación por el plagio y la citación o el asombro de poder trabajar todos de forma conjunta en el blog.

En la antepenúltima sesión, se presentó el último contenido teórico y práctico de la asignatura: las comunidades en red. Se abordaron aspectos como la conceptualización, su funcionalidad y uso: administradores, moderadores y miembros, y sus beneficios para divulgar información académica y científica. Esta parte de la sesión se aborda con metodología de lección magistral, mientras que su aplicabilidad a través de la herramienta Google+ se hace con sesiones prácticas, para explicar la 
creación de una comunidad con este recurso Web 2.0, aceptación de miembros, imagen que se muestra, enlazar contenidos, etc. Finalmente se les enseña la comunidad en red diseñada: Metic2017.

En las últimas sesiones, se les propone la tercera tarea y se les solicita que se unan a la comunidad creada por la docente, realizando la presentación del grupo y del blog, así como el enlace del blog para su difusión entre grupos y entre líneas.

En definitiva, mediante la comunidad Metic2017 se difunden y transmiten a la sociedad los recursos diseñados para dar respuesta al supuesto inicial planteado. Hay que mencionar que, de forma mayoritaria, las dificultades surgían de la aplicación Plotagon Education, concretamente de la conversión a formato vídeo, la extracción de la composición para difundirla en el blog, así como, la publicación de los comentarios en la comunidad.

\subsection{DESARROLLO DE LA EXPERIENCIA EN LA PRÁCTICA INTERDISCIPLAR “POESÍA INFANTIL” EN LAS ASIGNATURAS CCEE, FORMLIT Y EMADT}

La integración de los aprendizajes es uno de los valores añadidos a la formación del alumnado universitario, pues permite fortalecer las enseñanzas y dotarlas de funcionalidad de cara a la labor profesional. Por este motivo, se optó por conformar, para la experiencia, una práctica conjunta entre tres asignaturas, al poder establecer entre ellas relaciones muy complementarias y oportunas en la formación inicial de maestros y maestras: la educación en valores, su expresión a través de la experiencia estética, en concreto por medio de la poesía infantil, y su transmisión con la elaboración de material audiovisual como recurso tecnológico educativo.

En primer lugar, al comienzo del cuatrimestre, se presentó la experiencia al grupo 1 y al grupo 2 de estudiantes del Grado, enmarcada en el Plan de Innovación para el Curso 2016/2017 de la Universidad de Córdoba, en la modalidad 2. Programa de Formación para la innovación Docente, con la finalidad de contextualizar las sesiones de formación teóricapráctica básica que se llevarían a cabo en cada materia. Además, se explicó el trabajo a realizar desde las asignaturas implicadas en él, de manera que, en grupos de cuatro o cinco, tendrían que diseñar y elaborar material multimedia (vídeo en formato mp4), dirigido al alumnado de la etapa de Educación Primaria, con el cual se diera a conocer una selección de poemas tradicionales infantiles, así como poemas de autor en español y dirigidos a la infancia, que transmitieran valores.

Cabe mencionar que por las peculiaridad de la modalidad del Plan de Innovación y la distribución de la labor docente en el Centro, la asignatura FORMLIT solo pudo participar en el grupo 1 del Grado en Educación Primaria, realizando algunas de las metodologías, de manera adicional, para el grupo 2 del Grado.

La metodología utilizada se basó en la lección magistral sobre los contenidos literarios, el análisis y reflexión sobre los valores, así como sobre los elementos morfológicos del lenguaje audiovisual. A continuación, de manera simultánea, se realizaron, por un lado, talleres de tratamiento de imagen y de edición de vídeo, y un laboratorio de poesía para el conocimiento de la lírica infantil, tradicional y de autor, que proporcionó un escenario para la creación de poemas infantiles, a tenor de su tendencia genérica y su patrón estructural.

Terminado este primer periodo de aprendizaje, se indicaron las directrices necesarias para la elaboración del material. De manera secuenciada, se completarían dos fases, una de carácter individual y otra de carácter grupal (equipos de cuatro o cinco estudiantes).

En la primera (individual), se le requirió, a cada alumno y alumna, desde la asignatura de FORMLIT, la búsqueda y elección de dos poemas, uno perteneciente a la lírica tradicional infantil y otro de autor, en español y dirigido a la infancia, que representaran o trasmitieran valores apropiados para el alumnado de Educación Primaria, destinatarios de los mismos. A través de tutorías personalizadas se dirigió el proceso de selección, para evitar reiteraciones y asegurar la calidad de los poemas, la pertinencia a la tipología exigida y la adecuación a los receptores.

Una vez analizados y seleccionados los poemas, se procedió a la revisión y elección de los valores que transmitían, desde la asignatura de CCEE. Para poder realizar esta acción, se necesitó desarrollar unas sesiones previas de contextualización general de valores que pueden existir en las producciones infantiles y que los docentes deben conocer y saber explicar a su alumnado. En las citadas sesiones de contextualización y elección de los valores fue preciso llevar a cabo una criba por parte del grupo de trabajo, para evitar las repeticiones en el material multimedia a crear.

Realizadas las acciones de concreción del contenido del material a realizar, se procedió a la implementación de la $2^{\mathrm{a}}$ fase del trabajo, de carácter grupal, en la cual el alumnado planificó y grabó un vídeo educativo. Para ello, se atendió a la morfología y sintaxis del lenguaje audiovisual, la localización de los escenarios, selección del atrezo, acorde a la naturaleza de los textos a trabajar, y la disponibilidad de dispositivos, así como las herramientas tecnológicas para su registro y edición.

El material atendió a la estructura propia de los recursos educativos y de los trabajos académicos. Debía comenzar con una portada, con el título y los datos de los componentes del grupo, seguido de la explicación del valor o valores contenidos en los textos, la lectura de las obras y una reflexión grupal, a modo de conclusión, sobre los aprendizajes.

Debido al peso de los archivos generados, la entrega del material se formalizó vía telemática, a través de su envió por la plataforma We'Transfer. 
La evaluación se llevó a cabo de manera conjunta por el profesorado responsable de las asignaturas. Para valorar el logro de las competencias se utilizó una lista de control conformada por los criterios asociados a la definición de los valores, el orden y la claridad en la estructura del vídeo (guion de realización), utilización de los elementos audiovisuales, aspectos estéticos del contexto, tonalidad y modulación de la voz, claridad al hablar y vocalización, dominio del espacio y expresión corporal.

A su vez, al finalizar la práctica solicitada, como se ha mencionado, se implementó un cuestionario para recabar la opinión del alumnado con respecto a la metodología, al logro de los objetivos de la innovación y al nivel de implicación en el proceso de elaboración del material.

\section{RESULTADOS OBTENIDOS}

Los resultados se van a describir, igual que en apartados anteriores, teniendo en cuenta que la experiencia se ha realizado en cinco asignaturas, dos de ellas con prácticas propias de su disciplina y otras tres asociadas en una práctica interdisciplinar. Los resultados obtenidos, mediante la encuesta de satisfacción de la experiencia, evidencian el nivel de satisfacción del alumnado y su grado de implicación en la práctica.

\subsection{DESARROLLO RESULTADOS EN LA ASIGNATURA MPDI}

En relación al tipo de práctica implementada la valoración del alumnado ha sido bastante buena. No obstante, una debilidad que ha presentado esta práctica ha sido el hecho de que el alumnado no pudiera elegir el tipo de texto, sino que fuese el que se le adjudicara de manera aleatoria. Cabe destacar que no todos los textos literarios tenían el mismo grado de dificultad y que no a todos los alumnos les gustase el texto. Así mismo, en cuanto al agrupamiento hubiera sido más conveniente trabajar en parejas, de esta manera aquel alumnado que se hubiera sentido retraído para realizar la dramatización, hubiera estado más arropado y motivado. Tan solo se constituyó una pareja que trabajó el texto literario de forma conjunta, mientras que el resto debió realizarlo de manera individual.

El total de estudiantes que aprueban la práctica, en primera convocatoria, es del $94 \%$ del total de matriculados, no superando el trabajo el 6\% (tan solo un alumno no se presenta a la convocatoria de examen).

\subsection{RESULTADOS EN LA ASIGNATURA METTIC}

Los resultados académicos obtenidos han sido numerosos, dado que se deben valorar, por un lado, las calificaciones obtenidas en la prueba escrita y, por otro, las tres tareas solicitadas. En referencia a estas últimas se puede extraer de la evaluación lo siguiente:

- Tarea 1. Búsquedas con una ponderación del $20 \%$ en la calificación de los contenidos prácticos. En la línea A (o bilingüe) el $87 \%$ de los estudiantes han obtenido sobresaliente y el 10\% notable, los restantes aprobado. Referente a la línea B el 5\% han aprobado y el 70\% han conseguido sobresaliente, mientras que el resto notable. Finalmente en la línea $\mathrm{D}$, el $85 \%$ de los estudiantes han obtenido sobresaliente en esta tarea. El 7\% solo han aprobado. Y el restante $8 \%$ una calificación de notable.

- Tarea 2. Blog y recurso audiovisual (ponderación del 60\%). Las calificaciones han oscilado entre el 80\% y el 88\% de sobresalientes y el 20\% y el $12 \%$ de notable (según línea).

- Tarea 3. Comunidad en red (peso 20\%). En ella, casi el 100\% de todas las líneas ha obtenido sobresaliente como calificación grupal.

Estos materiales diseñados por el alumnado se encuentran depositados y alojados en la comunidad en red, creada mediante Google+, denominada Metic2017 (https:/plus.google.com/u/0/communities/111066400085026240872)

En relación a las calificaciones teóricas obtenidas en esta asignatura, en la primera convocatoria, han sido las siguientes:

- Línea A: el $94.55 \%$ del alumnado han superado el examen, el $2.725 \%$ no se han presentado y los restantes han suspendido.

- Línea B: el 84.06\% han superado el examen, mientras que el $15.94 \%$ han suspendido, del $97.18 \%$ presentados. El $2.82 \%$ no se han presentado.

- Línea D: el 96.08\% han aprobado la prueba escrita y el 3.92\%, de un 91.07\% de presentados, no la han superado. Mientras que el $8.93 \%$ no han acudido a la prueba en primera convocatoria.

Los datos empíricos obtenidos sobre la satisfacción con la experiencia se exponen a continuación. Atendiendo a la valoración del alumnado, la modalidad más apreciada son las sesiones magistrales de búsqueda de información ( $\mathrm{M}=3.73$, $\mathrm{SD}=0.646)$, entorno Google y sus aplicaciones $(\mathrm{M}=3.61, \mathrm{SD}=0.506)$ y $\operatorname{los}$ blogs $(\mathrm{M}=3.66, \mathrm{SD}=0.522)$. 
Tabla 1. Medias y desviaciones típicas de la valoración de los estudiantes en las modalidades empleadas en la asignatura y las acciones de innovación. ITEMS

\begin{tabular}{|c|c|c|c|}
\hline & & & \\
\hline Modalidad Magistral en la Búsqueda de información & 176 & 3.59 & 0.540 \\
\hline Modalidad Magistral en el entorno Google & 176 & 3.58 & 0.527 \\
\hline Modalidad Magistral sobre los Blogs & 176 & 3.57 & 0.528 \\
\hline Modalidad Magistral sobre las Comunidades en Red & 176 & 3.23 & 0.646 \\
\hline Sesiones Prácticas en la Búsqueda de información & 176 & 3.73 & 0.478 \\
\hline Sesiones Prácticas en el entorno Google y sus aplicaciones & 176 & 3.61 & 0.506 \\
\hline Sesiones Prácticas sobre Blogs & 176 & 3.66 & 0.522 \\
\hline Sesiones Prácticas sobre la aplicación Plotagon & 176 & 3.25 & 0.732 \\
\hline Sesiones Prácticas sobre Movie Maker & 176 & 2.82 & 0.873 \\
\hline Sesiones Prácticas sobre las comunidades en red & 176 & 3.24 & 0.684 \\
\hline Las tutorías o asistencias grupal sobre aspectos puntuales & 176 & 3.44 & 0.696 \\
\hline
\end{tabular}

Nota. La escala de intervalos propuesta corresponde a 1 significaba nada, 2 es poco, 3 es bastante y 4 es mucho.

La modalidad peor valorada fue las sesiones prácticas con Movie Maker, quizás por ser la herramienta que más conocen.

Tabla 2. Medias y desviaciones típicas de la estimación de los alumnos en relación a algunos aspectos de su aprendizaje.

ITEMS

Creo que mi competencia digital ha mejorado

Creo que mi competencia informacional ha mejorado

Creo que mi competencia tecnológica o informática ha aumentado

La gestión realizada del tiempo ha logrado una obtención progresiva de las tareas propuestas

La atención en las clases teóricas ha conseguido la adquisición de nuevos aprendizajes en relación a las TIC.

Nota. La escala de intervalos propuesta corresponde a 1 significaba totalmente en desacuerdo; 2 , en desacuerdo; 3 , indiferente; 4 , de acuerdo y 5 . totalmente de acuerdo.

El alumnado de primer curso, que ha participado en la experiencia, en la asignatura de "Métodos de investigación educativa y aplicaciones de las TIC" (impartida en el $2^{\circ}$ cuatrimestre en la FCE), están de acuerdo que su competencia digital ha mejorado $(\mathrm{M}=4.34, \mathrm{SD}=0.608)$ y que su atención en las clases ha conseguido la adquisición de nuevos aprendizajes en relación con las TIC $(\mathrm{M}=4.42, \mathrm{SD}=0.659)$. Por el contrario, la afirmación peor estimada ha sido la relativa a la gestión del tiempo $(\mathrm{M}=4.19, \mathrm{SD}=0.649)$.

Tabla 3. Medias y desviaciones típicas de la percepción de los estudiantes relativa a la implicación en la consecución de los objetivos.

ITEMS N N

Ob1. Reflexionar sobre el contexto en el que se desarrolla la Sociedad de la Información y el 176

Conocimiento.

Ob2. Considerar las TIC como recursos empleados en mi futura profesión docente.

Ob3. Analizar la amplitud e implicaciones que subyacen en el desarrollo de la competencia digital, en

concreto en la competencia informacional y competencia tecnológica o informática.

Ob4. Contemplar la relevancia de las estrategias de búsqueda de información (saber buscar,

monitorizar y salvaguardar) tanto en Internet como en la Biblioteca digital de la Universidad, que permiten el inicio a la gestión de la información.

Ob5. Conocer el entorno y, algunas de las aplicaciones más empleadas en la etapa de primaria, de Google.

Ob6. Atender de forma particular la aplicación Drive como recurso para el aprendizaje cooperativo.

Ob7. Conocer y utilizar educativamente, comprendiendo, analizando y reflexionando, sobre algunas

herramientas Web 2.0 y materiales multimedia.

Ob8. Utilizar el lenguaje audiovisual para comunicar de forma eficaz y comprensiva.

Ob9. Estimular la creación, diseño y uso de los blog como recursos para difundir información.

Ob10. Conocer y emplear Google+ como recurso para difundir, colaborar y transferir aportaciones

educativas y científicas con una comunidad.

Ob11. Apreciar nuevos entornos de relación virtual con carácter científico o académico.

Ob12. Reflexionar y analizar el tratamiento de los contenidos que se imparten en la etapa de primaria.

Ob13. Analizar la utilidad de los medios tecnológicos (en concreto los audiovisuales) como

potenciadores del patrimonio cultural y la tradición oral en la poesía infantil.

\begin{tabular}{ccc}
$\mathbf{N}$ & $\mathbf{M}$ & $\mathbf{S D}$ \\
\hline 176 & 4.34 & 0.608 \\
176 & 4.25 & 0.615 \\
\hline 176 & 4.22 & 0.675 \\
\hline 176 & 4.19 & 0.649 \\
176 & 4.42 & 0.659
\end{tabular}

Nota. La escala de intervalos propuesta corresponde a 1 significaba nada; 2 es poco; 3 es bastante; y, 4 es mucho.

Los estudiantes han percibido que su implicación (motivación y/o participación) mayor ha sido en los objetivos de "Considerar las TIC como recursos empleados en mi futura profesión docente" ( $\mathrm{M}=3.86, \mathrm{SD}=0.430)$; "Conocer el entorno 
y, algunas de las aplicaciones más empleadas en la etapa de primaria, de Google" $(\mathrm{M}=3.64, \mathrm{SD}=0.528)$; "Atender de forma particular la aplicación Drive como recurso para el aprendizaje cooperativo" $(\mathrm{M}=3.59, \mathrm{SD}=0.596)$; "Estimular la creación, diseño y uso de los blog como recursos para difundir información" ( $\mathrm{M}=3.75, \mathrm{SD}=0.435)$; y, "Analizar la utilidad de los medios tecnológicos (en concreto los audiovisuales) como potenciadores del patrimonio cultural y la tradición oral en la poesía infantil" $(\mathrm{M}=3.53, \mathrm{SD}=0.560)$. Mientras que exponen menor implicación en "Reflexionar sobre el contexto en el que se desarrolla la Sociedad de la Información y el Conocimiento" ( $\mathrm{M}=3.12, \mathrm{SD}=0.597)$.

\subsection{RESULTADOS EN LA PRÁCTICA INTERDISCIPLINAR “POESÍA INFANTIL” EN LAS ASIGNATURAS CCEE, FORMLIT Y EMADT}

A nivel académico, los resultados han sido muy satisfactorios. De los 101 estudiantes matriculados el $20 \%$ aprobó, el $38,6 \%$ obtuvo una calificación de notable y el 31,7\% tuvieron un sobresaliente. Solo el 6,9\% no superó la práctica y el 2\% no se presentó en primera convocatoria.

Por otro lado, los datos obtenidos del estudio realizado con la intención de comprobar la funcionalidad y consecución de los objetivos planteados en la experiencia, durante el proceso de elaboración y edición del material multimedia, en los tres bloques que lo componen, ostentan resultados por encima del valor medio.

En lo relativo a la metodología utilizada, el alumnado valora positivamente las horas lectivas cedidas para el trabajo en grupo $(\mathrm{M}=3.87, \mathrm{SD}=1.036)$, las sesiones de revisión de los poemas $(\mathrm{M}=3.73, \mathrm{SD}=1.029)$ y las sesiones sobre los aspectos teóricos del Lenguaje audiovisual $(\mathrm{M}=3.71, \mathrm{SD}=0.942)$. Por el contrario, los elementos con valoraciones más bajas coinciden con los ítems relativos a la asignatura FORMLIT, ya que como se comentó en la descripción de la experiencia, no se pudo llevar a cabo en ambos grupos aula.

Tabla 4. Medias y desviaciones típicas de la valoración de los estudiantes en las modalidades utilizadas entorno a la práctica. ITEMS

\begin{tabular}{|c|c|c|c|}
\hline ITEMS & $\mathbf{N}$ & $\mathbf{M}$ & SD \\
\hline Lección magistral (sesiones de explicación del bloque temático sobre valores). & 101 & 3.51 & 0.945 \\
\hline Lección magistral (sesiones de explicación de los aspectos teóricos sobre Lenguaje audiovisual). & 101 & 3.71 & 0.942 \\
\hline Lección magistral (sesiones de explicación del bloque temático sobre la Lírica infantil. & 101 & 3.37 & 0.997 \\
\hline Laboratorio de poesía infantil. & 55 & 3.45 & 0.835 \\
\hline Revisión de la selección de poemas. & 101 & 3.73 & 1.029 \\
\hline Revisión de la adecuación temática de los valores. & 98 & 3.67 & 1.003 \\
\hline Taller sobre edición de vídeo para la creación de material multimedia. & 101 & 3.54 & 1.221 \\
\hline Consultas de asesoramiento on-line (correos electrónicos). & 100 & 3.55 & 1.158 \\
\hline Horas lectivas para la realización del trabajo en grupo (Educación Mediática). & 101 & 3.87 & 1.036 \\
\hline
\end{tabular}

Horas lectivas para la realización del trabajo en grupo (Educación Mediática).

Nota. La escala de intervalos propuestas corresponde a 1 significaba nada; 2 es poco; 3 es algo; 4 es mucho y 5 es mucho.

Con respecto al logro de los objetivos planteados, destacan los valores concedidos al ítem sobre la edición de material multimedia susceptible de ser utilizado en el aula $(\mathrm{M}=3.89, \mathrm{SD}=0.871)$, a los ítems "Mostrar a la sociedad los valores humanos contenidos en estas obras y la importancia de la experiencia estética" ( $\mathrm{M}=3.86, \mathrm{SD}=0.949)$ y "Sensibilizar a los futuros docentes en el compromiso social de la Universidad con la difusión de la cultura española, como como elemento de transferencia de conocimiento $(\mathrm{M}=3.82, \mathrm{SD}=0.984)$. A su vez, teniendo presente la naturaleza de la experiencia, resultan interesantes los datos que presentan los ítems "Colaborar en el conocimiento y difusión, desde las aulas de la enseñanza obligatoria (Infantil y Primaria), de la poesía popular infantil, patrimonio de nuestra cultura" $(\mathrm{M}=3.72, \mathrm{SD}=0.850)$ y "Formar al alumnado de los Grados participantes en las competencias transversales (TIC y emprendimiento) a través de actividades académicamente dirigidas multidisciplinares" $(\mathrm{M}=3.71, \mathrm{SD}=0.829)$. Los aspectos que muestran resultados más bajos son "Conocer la Lírica infantil tradicional y de autor para colaborar, como futuros docentes, en su transmisión y difusión" ( $\mathrm{M}=3.42, \mathrm{SD}=1.042)$ e "Iniciar al alumnado de los Grados de Educación Infantil y Educación Primaria en la creación de textos poéticos infantiles." ( $\mathrm{M}=3.46, \mathrm{SD}=0.911)$, aunque hay que incidir en que estos están por encima de la media.

Tabla 5. Medias y desviaciones típicas de la estimación de los alumnos en relación a en la consecución de los objetivos de la experiencia.

\begin{tabular}{|c|c|c|c|}
\hline ITEMS & $\mathbf{N}$ & $\mathbf{M}$ & SD \\
\hline $\begin{array}{l}\text { Conocer la Lírica infantil tradicional y de autor para colaborar, como futuros docentes, en su } \\
\text { transmisión y difusión. }\end{array}$ & 101 & 3.42 & 1.042 \\
\hline $\begin{array}{l}\text { Editar material multimedia que pueda ser utilizado en las aulas de la etapa de Educación Infantil y } \\
\text { Educación Primaria y que contribuya a recuperar, transmitir y difundir la poesía infantil. }\end{array}$ & 101 & 3.89 & 0.871 \\
\hline $\begin{array}{l}\text { Colaborar en el conocimiento y difusión, desde las aulas de la enseñanza obligatoria (Infantil y } \\
\text { Primaria), de la poesía popular infantil, patrimonio de nuestra cultura. }\end{array}$ & 101 & 3.72 & 0.850 \\
\hline $\begin{array}{l}\text { Iniciar al alumnado de los Grados de Educación Infantil y Educación Primaria en la creación de textos } \\
\text { poéticos infantiles. }\end{array}$ & 101 & 3.46 & 0.911 \\
\hline $\begin{array}{l}\text { Mostrar a la sociedad los valores humanos contenidos en estas obras y la importancia de la experiencia } \\
\text { estética. }\end{array}$ & 101 & 3.86 & 0.949 \\
\hline Formar al alumnado de los Grados participantes en las competencias transversales (TIC y & 101 & 3.71 & 0.829 \\
\hline
\end{tabular}


emprendimiento) a través de actividades académicamente dirigidas multidisciplinares.

Sensibilizar a los futuros docentes en el compromiso social de la Universidad con la difusión de la

cultura española, como como elemento de transferencia de conocimiento.

$101 \quad 3.82$

0.984

Nota. La escala de intervalos propuestas corresponde a 1 significaba nada; 2 es poco; 3 es algo; 4 es mucho y 5 es mucho.

Por último, en general, el alumnado está satisfecho con su participación en los distintos momentos y acciones de esta experiencia. Reconoce su implicación en la "Búsqueda y selección del poema de un autor español o de habla hispana" $(M=4.01, S D=0.789)$, aunque se posiciona con el valor más bajo $(\mathrm{M}=3.74, \mathrm{SD}=0.917)$ respecto a su implicación en las clases teóricas sobre la temática perteneciente Lírica infantil. También valora muy positivamente su participación en la "Aportación de ideas para el montaje audiovisual del material multimedia a grabar" $(\mathrm{M}=4.02, \mathrm{SD}=0.866)$.

Tabla 6. Medias y desviaciones típicas de la percepción de los estudiantes relativa a la implicación en las distintas fases de elaboración del material solicitado. ITEMS

\begin{tabular}{|c|c|c|c|}
\hline 11EMS & $\mathbf{N}$ & M & SD \\
\hline Atención y participación en las clases teóricas sobre la temática perteneciente al Lenguaje audiovisual. & 101 & 3.92 & 0.808 \\
\hline Atención y participación en las clases teóricas sobre la temática perteneciente a los Valores. & 101 & 3.94 & 0.835 \\
\hline Atención y participación en las clases teóricas sobre la temática perteneciente Lírica infantil. & 100 & 3.74 & 0.917 \\
\hline Búsqueda y selección del poema perteneciente a la Lírica tradicional. & 101 & 3.92 & 0.891 \\
\hline Búsqueda y selección del poema de un autor español o de habla hispana. & 99 & 4.01 & 0.789 \\
\hline Análisis de los valores objeto de reflexión a través de los poemas. & 101 & 3.92 & 0.808 \\
\hline Aportación de ideas para el montaje audiovisual del material multimedia a grabar. & 101 & 4.01 & 0.866 \\
\hline
\end{tabular}

Nota. La escala de intervalos propuestas corresponde a 1 significaba nada; 2 es poco; 3 es algo; 4 es mucho y 5 es mucho.

\section{UTILIDAD/ANÁLISIS}

Con esta experiencia se ha fomentado el protagonismo del alumnado en su proceso formativo, poniendo de manifiesto las competencias logradas a través de ella, junto a las adquiridas en cursos anteriores. Además, se ha puesto especial interés en el potencial que esta tiene para la formación en competencias transversales (competencias comunicativas bilingüe, digital, intercultural y social).

Por la naturaleza y simplicidad de las acciones que el alumnado ha desarrollado, por la posibilidad de trabajar conjuntamente varias disciplinas, así como por el tipo de materiales que se pueden realizar, esta práctica es idónea para implementarla, no solo con alumnado universitario del Grado de Educación Primaria, sino también con el alumnado de $2^{\circ}$ y $3^{\circ}$ ciclo de la etapa de Educación Primaria y primer ciclo de la etapa de Educación Secundaria Obligatoria.

\section{CONCLUSIONES/DISCUSIÓN}

El mundo educativo se ha visto, en los últimos tiempos, mediado por las producciones y la utilización de las programaciones editoriales. Sin embargo, sería conveniente la convivencia entre estas y la generación de recursos didácticos originales, personalizados a las necesidades educativas del alumnado. Por ello, desde las enseñanzas universitarias en las que se forman a los futuros docentes, es importante fomentar la capacidad de emprendimiento para la creación de estos materiales. No se trata de adiestrarlos en lo que ya existe y se usa en el aula, si no ofrecerles los conocimientos, las herramientas y las estrategias que posibilitan la integración de nuevas producciones en las planificaciones didácticas.

Además, es primordial diseñar prácticas que evidencien, a lo largo de la formación de los maestros y maestras, la funcionalidad de los aprendizajes y cómo estos ayudan a relacionar la teoría con la práctica. Con ello, se añade un componente motivacional que predispone al alumnado a la implicación en el desarrollo de las propuestas. A su vez, gracias a la cooperación entre profesorado de diversas áreas de conocimiento, el alumnado adquiere una serie de competencias ligadas a las disciplinas en cuestión y a las denominadas competencias transversales (competencias comunicativas, digitales, bilingües y sociales), que son las que fomentan la reflexión sobre la lengua materna y/o lengua extranjera, sobre los valores y garantizan el uso de las nuevas tecnologías.

Por otro lado, se ha podido observar, en las destrezas evidenciadas en el alumnado de cuarto curso, que los aprendizajes adquiridos a lo largo de los cuatro años de carrera se movilizan en la construcción de materiales educativos, lo que asegura la calidad del proceso formativo.

Para el alumnado del Grado de Educación Primaria, la elaboración de trabajos que nazcan de la búsqueda y selección de información, que se sustenten en el análisis de la pertinencia educativa y que, a su vez, puedan colaborar en el conocimiento y la difusión de aspectos propios de nuestra cultura, hace que tome relevancia la responsabilidad que conlleva la labor para la que se prepara en esta etapa formativa. La aportación de esta experiencia al reconocimiento de la lírica infantil, tan poco apoyada editorialmente, y, especialmente a la conservación y difusión de la poesía tradicional se hace notable en este sentido.

Igualmente, las acciones educativas implementadas a través de la innovación, fomentan la sensibilización del alumnado de los Grados en el compromiso social de la institución universitaria con la difusión de la literatura y, con ello, de la cultura española, como elemento de transferencia de conocimiento en una sociedad multilingüe e intercultural como la actual. 
Para finalizar, hay que resaltar la necesidad de potenciar en los diversos niveles educativos, desde edades tempranas hasta la universidad, los nuevos modelos de comunicación que emergen de la evolución tecnológica y sobre todo telemática, que proporcionan un vía para la transferencia de conocimiento y, por ende, el desarrollo de la sociedad en general. Todo ello gracias a la capacidad de difusión por las diversas plataformas, foros, portales, canales, etc., la pluralidad de lenguajes que combinan y el contexto que ofrecen para la cooperación.

\section{BIBLIOGRAFÍA}

1. Bazo, P., D’Angelo, E. y Valdés, M. Proyecto Plurilingüe: Notas para su construcción 2010. Recuperado de http://blog.educastur.es/equiposdirectivos/files/2010/11/proyecto-plurilingue.pdf

2. Cerrillo, P. y Sánchez Ortiz, C. (2012) De la oralidad a la escritura: Un camino de ida y vuelta en el Cancionero Popular Infantil. Olivar 2012;13(18):317-342. Recuperado de: http://www.memoria.fahce.unlp.edu.ar/art_revistas/pr.5837/pr.5837.pdf p.24

3. De Miguel, M. (2006) Metodologías de enseñanza y aprendizaje para el desarrollo de competencias. Orientaciones pare el profesorado universitario ante el Espacio Europeo de Educación Superior. Madrid: Alianza. I.S.B.N.-10: 848317-546-0 I.S.B.N.-13: 978-84-8317-546-0.

4. Durán, J. F. y Vega, M. R. (2013) Las Tecnologías de la Información y la Comunicación en las Facultades de Educación. Historia y Comunicación Social 2013;18:313-326. DOI: http://dx.doi.org/10.5209/rev_HICS.2013.v18.44245

5. Fernández-Batanero, J. M. y Torres-González, J. A. (2015) Actitudes docentes y buenas prácticas con TIC del profesorado de Educación Permanente de Adultos en Andalucía. Revista Complutense de Educación 2015;26:3349. DOI: http://dx.doi.org/10.5209/rev_RCED.2015.v26.43812

6. González, I., Marín, V. y Martín, M. A. (2015) El significado de la Universidad desde la mirada del alumnado. Indicadores para su evaluación. Revista Aula de Encuentro 2015;2(17): 33-58. Recuperado de http://revistaselectronicas.ujaen.es/index.php/ADE/article/view/2657

7. Johnson, L., Adams, S., Cummins, M., Estrada, V., Freeman, A. y Hall, C. (2016) NMC Horizon Report: 2016 Higher Education Edition. Austin, Texas: The New Media Consortium. ISBN 978-0-9968527-8-4.

8. Marín, V. y Reche, E. (2012) Universidad 2.0: Actitudes y aptitudes ante las TIC del alumnado de nuevo ingreso de la escuela universitaria de magisterio de la UCO. Pixet-Bit. Revista de Medios y Educación 2012;40:197-211.

9. López Tamés, R. (1990) Introducción a la literatura infantil. Murcia: Secretariado de Publicaciones. ISBN 84-7684205-8.

10. Mendoza, A. (2016) Didáctica de la Lengua y de la Literatura para Primaria. Madrid: Pearson Educación. ISBN: 9788420534558.

11. Sabariego, M. (2012) La investigación educativa: Génesis, Evolución y Características. En R. Bisquerra (coord.). Metodología de la investigación educativa. Madrid: La Muralla. ISBN: 84-7133-748-7.

12. Sabariego, M. y Bisquerra, R. (2012) Fundamentos metodológicos de la investigación educativa. En R. Bisquerra (coord.). Metodología de la investigación educativa. Madrid: La Muralla. ISBN: 84-7133-748-7.

13. Sánchez, S. (2010) Didáctica de la Educación en valores. En A. De la Herrán y J. Paredes (coords.). Didáctica General. La práctica de la enseñanza en educación infantil, primaria y secundaria. Aravaca, Madrid: MacGrawHill. ISBN: 978-84-481-6637-3. 